\title{
Epigenome-wide inheritance of cytosine methylation variants in a recombinant inbred population
}

\author{
Robert J. Schmitz, ${ }^{1,2,11}$ Yupeng He, 2,3,11 Oswaldo Valdés-López, ${ }^{4,12}$ Saad M. Khan, ${ }^{4,5}$ \\ Trupti Joshi, ${ }^{4,5,6,7}$ Mark A. Urich, ${ }^{2}$ Joseph R. Nery, ${ }^{2}$ Brian Diers, ${ }^{8}$ Dong Xu, ${ }^{4,5,6,7}$ \\ Gary Stacey, ${ }^{4,7,9,13}$ and Joseph R. Ecker ${ }^{1,2,10,13}$ \\ ${ }^{1}$ Plant Biology Laboratory, The Salk Institute for Biological Studies, La Jolla, California 92037, USA; ${ }^{2}$ Genomic Analysis Laboratory, The \\ Salk Institute for Biological Studies, La Jolla, California 92037, USA; ${ }^{3}$ Bioinformatics Program, University of California at San Diego, \\ La Jolla, California 92093, USA; ${ }^{4}$ Christopher S. Bond Life Sciences Center, University of Missouri, Columbia, Missouri 65211, USA; \\ ${ }^{5}$ Informatics Institute, University of Missouri, Columbia, Missouri 65211, USA; ${ }^{6}$ Department of Computer Science, University of \\ Missouri, Columbia, Missouri 65211, USA; ${ }^{7}$ National Center for Soybean Biotechnology, University of Missouri, Columbia, Missouri \\ 65211, USA; ${ }^{8}$ Department of Crop Sciences, University of Illinois, Urbana, Illinois 61801, USA; ${ }^{9}$ Divisions of Plant Science and \\ Biochemistry, University of Missouri, Columbia, Missouri 65211, USA; ${ }^{10}$ Howard Hughes Medical Institute, The Salk Institute for \\ Biological Studies, La Jolla, California 92037, USA
}

\begin{abstract}
Cytosine DNA methylation is one avenue for passing information through cell divisions. Here, we present epigenomic analyses of soybean recombinant inbred lines (RILs) and their parents. Identification of differentially methylated regions (DMRs) revealed that DMRs mostly cosegregated with the genotype from which they were derived, but examples of the uncoupling of genotype and epigenotype were identified. Linkage mapping of methylation states assessed from wholegenome bisulfite sequencing of 83 RILs uncovered widespread evidence for local methylQTL. This epigenomics approach provides a comprehensive study of the patterns and heritability of methylation variants in a complex genetic population over multiple generations, paving the way for understanding how methylation variants contribute to phenotypic variation.
\end{abstract}

[Supplemental material is available for this article.]

Phenotypic variation results from a combination of genetic variation, environment, and interactions among the two. The contribution of natural epigenetic variation to phenotypic variation still remains enigmatic due to the relatively few characterized natural epigenetic alleles (epialleles) (Bender and Fink 1995; Cubas et al. 1999; Manning et al. 2006; Rangwala et al. 2006; Hitchins et al. 2007; Woo et al. 2007; Becker et al. 2011; Schmitz et al. 2011; Durand et al. 2012). Epialleles are classified into three major groups, which are defined by their dependence on an underlying genetic variant (Richards 2006). Briefly, obligate epialleles are completely dependent on a genetic variant, whereas pure epialleles are maintained independently of genetic variants. The dependence on genetic variants for the third group, facilitated epialleles, breaks down because the genetic variant can influence the epiallelic state but not as reliably as they do for obligate epialleles (Richards 2006).

In Arabidopsis thaliana, there is extensive evidence for the involvement of epialleles in creating phenotypic diversity (Johannes et al. 2009; Reinders et al. 2009; Roux et al. 2011). Outside of Arabidopsis thaliana, these are most evident for the peloric, colorless non-ripening, and $B^{\prime}$ epialleles from Linaria vulgaris, Solanum lycopersicum, and Zea mays, respectively (Patterson et al. 1993; Cubas

\footnotetext{
${ }^{11}$ These authors contributed equally to this work.

12 Present address: Laboratorio de Bioquímica, Facultad de Estudios Superiores Iztacala, Universidad Nacional Autónoma de México, Tlalnepantla, Estado De México, Mexico 54090.

${ }^{13}$ Corresponding authors

E-mail ecker@salk.edu

E-mail staceyg@missouri.edu

Article published online before print. Article, supplemental material, and publication date are at http://www.genome.org/cgi/doi/10.1101/gr.152538.112.
}

et al. 1999; Manning et al. 2006). Still, these are rare events and appear to be the exception rather than the rule. Work in Arabidopsis thaliana has led to the most comprehensive analyses of natural epigenetic variation and uncovered a variety of modes to the formation of epialleles (Schmitz and Ecker 2012). These include genetic variants that can exert their influence on epiallelic states both locally and distantly to other chromosomes (Bender and Fink 1995; Rangwala et al. 2006; Woo et al. 2007; Durand et al. 2012; Schmitz et al. 2013).

The RNA-directed DNA methylation pathway (RdDM) (for review, see Law and Jacobsen 2010) provides a molecular basis for the formation and maintenance of epiallelic states of many of the identified epialleles in Arabidopsis and likely other flowering plant species. This pathway generates a feedback loop between small RNAs (smRNAs) and DNA methylation that represses gene expression and enables propagation of epiallelic states through both mitotic and meiotic cell divisions. The presence of smRNAs also provides sequence-specific guides that facilitate silencing at distant loci, even on different chromosomes.

Because most characterized epialleles contain distinct molecular signatures, usually smRNAs in combination with DNA methylation, it is possible to systematically determine how extensive natural epigenomic variation is in the wild. Pioneering efforts using epigenomic techniques (for review, see Schmitz and Zhang 2011) revealed extensive natural variation in methylation of gene bodies compared to smRNA-associated transposon and repetitive sequences between two accessions of Arabidopsis thaliana (Vaughn et al. 2007; Zhang et al. 2008). Similar epigenomic approaches in maize uncovered hundreds of differentially methylated regions (DMRs), some of which were subsequently found unlinked to genetic variants using near isogenic lines derived from the two profiled 
parental lines revealing the presence of heritable pure epialleles (Eichten et al. 2011).

A major challenge in understanding natural epigenetic variation is determining the dependence of methylation variants on genetic variants. Recent studies addressed one aspect of this challenge by using a population of mutation accumulation lines (Shaw et al. 2000), which reduced genetic variation to the spontaneous mutation rate (Ossowski et al. 2010), enabling a better understanding of pure epigenetic variation. These studies uncovered single methylation polymorphisms (SMPs) occurring at a much higher rate than DNA mutations and found that they primarily occurred in gene bodies (Becker et al. 2011; Schmitz et al. 2011). Larger regions of differential DNA methylation that resembled loci targeted by RdDM were also identified and some were even found to affect gene expression levels, although the rate of occurrence of these types of DMRs was similar to the spontaneous DNA mutation rate (Becker et al. 2011; Schmitz et al. 2011). Therefore, it is clear that natural epigenetic variation can be uncoupled from genetic variation in the laboratory, but in nature, these two types of variants coevolve.

Soybean (Glycine max L. merr.) is a major crop providing an important source of protein and oil. A high-quality reference soybean genome is available (Schmutz et al. 2010), which supports that this plant has experienced at least two polyploid events, the most ancient being 59 Mya. Soybean is considered an allopolyploid (Gill et al. 2009), which resulted from the merger of two genomes that diverged $\sim 13$ Mya and reunited $\sim 5-10$ Mya when the genus Glycine was formed (Doyle et al. 2003; Straub et al. 2006; Innes et al. 2008; Stefanovic et al. 2009). Roughly $75 \%$ of all soybean-coding sequences are present in two or more copies in the genome. Therefore, to understand the role of DNA methylation in this species and its impact on gene expression, we sequenced genomes, DNA methylomes, and transcriptomes in the parents and RILs. This also enabled us to understand how DNA methylation patterns are established, inherited, and maintained as they segregate through a complex genetic population. The vast majority of identified DMRs cosegregated with the genetic background from which they were identified, which enabled population-wide identification of methylQTL for $>90 \%$ of the DMRs. Rare examples of DMRs were identified that did not show evidence for linkage to a particular genomic region, which could be indicative of pure epigenetic variants.

The findings of this study have broad implications for the fields of crop epigenomics, epigenetics, inheritance of methylation variants, and plant breeding. There is a growing interest about the potential role for epigenetics to explain phenotypic diversity that cannot be attributed to genetics in a variety of systems, but the evidence is still limiting at the population level. This study clearly demonstrates that the majority of methylation variants adheres to Mendelian modes of inheritance but also demonstrates rare examples of epigenetic variation that do not follow the standard laws of inheritance.

\section{Results}

\section{Single-base resolution DNA methylome of Glycine max}

To understand the contribution of cytosine DNA methylation to the soybean genome, whole-genome bisulfite sequencing (MethylC-seq) (Lister et al. 2008) was performed on DNA isolated from leaves of the LD00-2817P germplasm (hereafter referred to as "LD"). In total, greater than 162 million 101-bp reads were sequenced that only aligned to unique regions of the genome, which represents approximately eightfold coverage per strand of the genome (Supplemental Table 1). Briefly, methylated cytosines were determined by applying a binomial test to data from reads covering each cytosine and using the unmethylated chloroplast genome as a control (see "Methods" for a more detailed description). The LD methylome contains 15,444,227 methylated CGs (mCG) (51\% of all CGs), 14,942,676 mCHG (39\% of all CHGs), and 13,628,219 $\mathrm{mCHH}(0.05 \%$ of all $\mathrm{CHHs})$, which represents a greater proportion of methylated cytosines compared with a recently reported DNA methylome for Arabidopsis thaliana (Fig. 1A; Schmitz et al. 2011). Of the cytosines that are methylated in the LD genome, there are almost equal numbers of $\mathrm{mCG}$ and $\mathrm{mCHG}$, which contrasts to the Arabidopsis thaliana methylome (Fig. 1B) and could indicate that RdDM targets a greater proportion of the soybean genome. Of the detected methylcytosines, the distribution of methylation levels at each site in each context was similar to the levels found in Arabidopsis thaliana, with the exception of $\mathrm{mCHG}$ (Supplemental Fig. 1A,B). In general, mCG and mCHG are methylated at higher levels as compared to $\mathrm{mCHH}$.

The distribution of $\mathrm{mCG}, \mathrm{mCHG}$, and $\mathrm{mCHH}$ sites genomewide revealed that gene-rich and transposon-poor euchromatic regions of each chromosome contain lower bulk methylation compared with the gene-poor and transposon-rich heterochromatic regions in the pericentromeres of the chromosomes (Fig. 1C; Supplemental Fig. 2A-F). Using previously published small RNA sequencing data (Tuteja et al. 2009), the relative abundance of 2124 nucleotide (nt) smRNAs were plotted along each chromosome, which revealed a higher density of 24-nt smRNAs in regions of the genome that contain abundant non-GC methylation (Fig. 1C; Supplemental Fig. 2G), as well as for 21-23-nt smRNAs (Supplemental Fig. 3).

Two whole genome duplications have occurred in the diploid ancestor of soybean, an early duplication $\sim 59$ million years ago (Mya) and a recent duplication $\sim 13$ Mya. (Schmutz et al. 2010). A comparison of the DNA methylation profiles between these duplicated regions revealed that younger sequences are more likely to contain greater amounts of DNA methylation typical of the RdDM pathway (Fig. 1C,D), indicating that these sequences are actively being silenced. In fact, CG, CHG, and CHH methylation was, on average, $\sim 10 \%, \sim 20 \%$, and $\sim 10 \%$ higher for recently duplicated regions, genes, or exons compared to early duplicated regions, respectively (Fig. 1D). The increase in DNA methylation of the recent duplications also was significantly differentially associated with distance from the pericentromeric regions when compared to the early duplication events (Mann-Whitney-Wilcoxon test, $P$-value < $2.2 \times 10^{-16}$ ) (Fig. 1E,F; Supplemental Fig. 4). Collectively, these results indicate that DNA methylation is one potential mechanism that plants use to cope with duplicated DNA and could potentially explain, in part, why the soybean genome contains greater amounts of DNA methylation compared to Arabidopsis thaliana.

\section{Patterns of DNA methylation in genes and transposons}

CG gene-body methylation appears to be conserved in plants and animals (Feng et al. 2010; Zemach et al. 2010b); and soybean is no exception (Fig. 2A), although its exact function is still unknown and not all genes in plant genomes contain CG gene-body methylation. The density of both $\mathrm{mCHG}$ and $\mathrm{mCHH}$ is lowest throughout the gene body when compared to transposons (Fig. 2A; Supplemental Fig. 5A-C), which is consistent with the lack of 24-nt smRNA-directed DNA methylation targeting most genes (Fig. 2B). The density of all three types of DNA methylation is higher at 
A

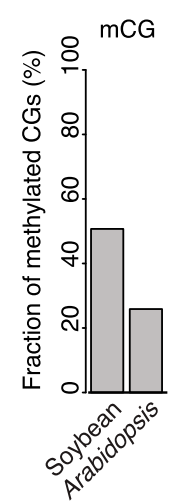

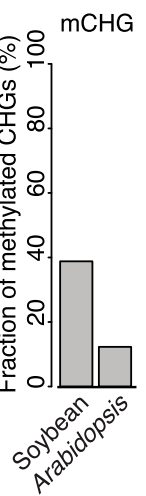

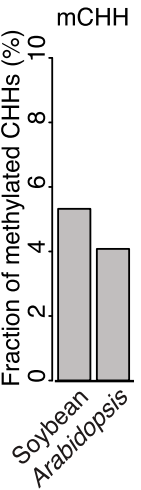

B

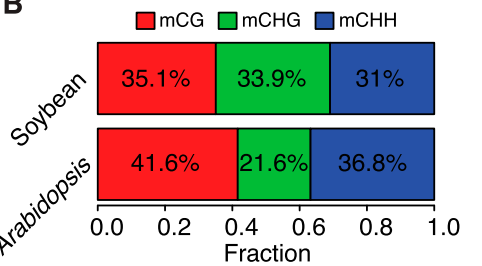

D

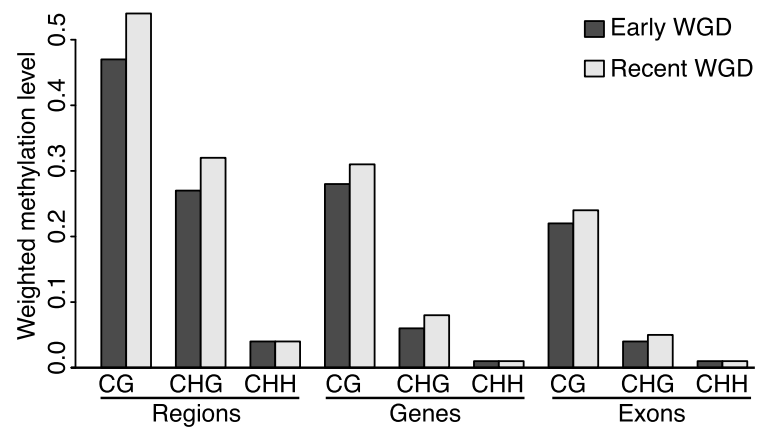

C

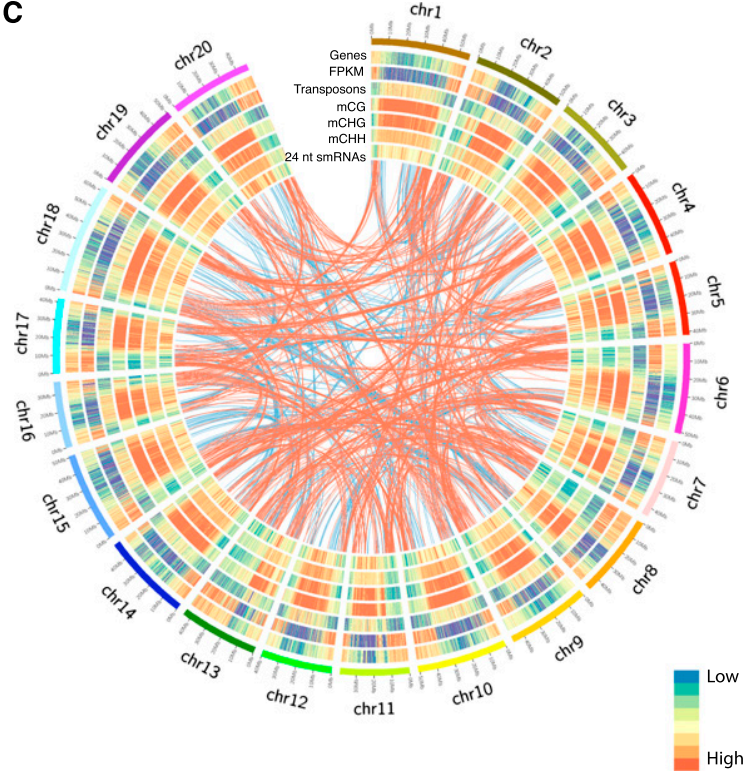

$\mathbf{E}$

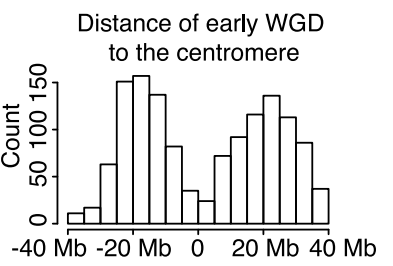

$\mathbf{F}$

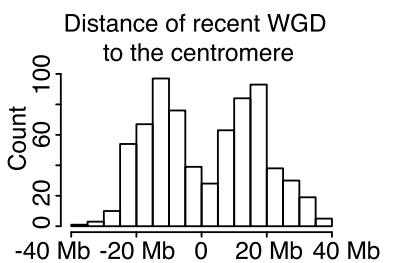

Figure 1. Characteristic features of the DNA methylomes between Arabidopsis thaliana and soybean. (A) Fraction of methylated cytosines for each context as a proportion of that context genome wide. (B) The DNA methylation present in the soybean methylome is highly enriched for $\mathrm{CHG}$ and $\mathrm{CHH}$ methylation. (C) A circle plot of gene density, transposon density, FPKMs, mCG, mCHG, mCHH, and 24-nt smRNAs for LD. (Red lines) Regions from the 59 Mya whole-genome duplication. (Blue lines) Regions from the recent whole-genome duplication 13 Mya. (D) Weighted methylation levels for early and recent whole-genome duplications (WGD) for the entire duplicated regions and genes and exons within those duplicated regions. $(E, F)$ Distance in Mb of duplicated regions from the centromeres.

sequences both upstream and downstream from the transcriptional start and stop sites increases (Fig. 2A; Supplemental Fig. 5D-F).

In contrast to CG gene-body methylation, transposons are targeted by the RdDM pathway, resulting in enriched levels of all types of DNA methylation and an abundance of 24-nt smRNAs (Fig. 2A,B; Supplemental Fig. 5G). Although the levels of all types of DNA methylation between the major classes of soybean retrotransposons (long terminal repeat [LTRs] and LINEs) and DNA transposons (terminal inverted repeats [TIRs] and helitrons) are similar, there are interesting characteristics that distinguish them from one another. The LTR and LINE retrotransposons contain the highest levels of DNA methylation near the actual repeat structures that define the $5^{\prime}$ and $3^{\prime}$ ends of retrotransposons (Fig. 2A), which coincides with the location containing the greatest abundance of 24-nt smRNAs (Fig. 2B; Supplemental Fig. 6A,B). This contrasts to the DNA methylation levels at the $5^{\prime}$ and $3^{\prime}$ ends of TIR transposons, which are depleted relative to the transposon bodies where 24-nt smRNAs are most abundant (Fig. 2B; Supplemental Fig. 6C). The higher density of methylation upstream and downstream from LTRs also distinguishes them from the other classes of transposons (Fig. 2A), which reflects their distribution along the chromosomes (Fig. 2C). LTR transposons are located in the heterochromatic pericentromeres, whereas TIRs and LINEs are located throughout the chromosome arms (Fig. 2C).

\section{Effects of DNA methylation on gene expression}

To better understand the role of DNA methylation and its association with gene expression, RNA was isolated from LD leaves and RNA-seq data were generated, aligned, and quantified (Methods; Supplemental Table 2). The levels of CG gene-body methylation were positively correlated with gene expression levels (Fig. 3A), whereas the levels of $\mathrm{CHG}$ or $\mathrm{CHH}$ methylation in gene bodies were negatively associated with gene expression levels (Fig. 3B,C), 

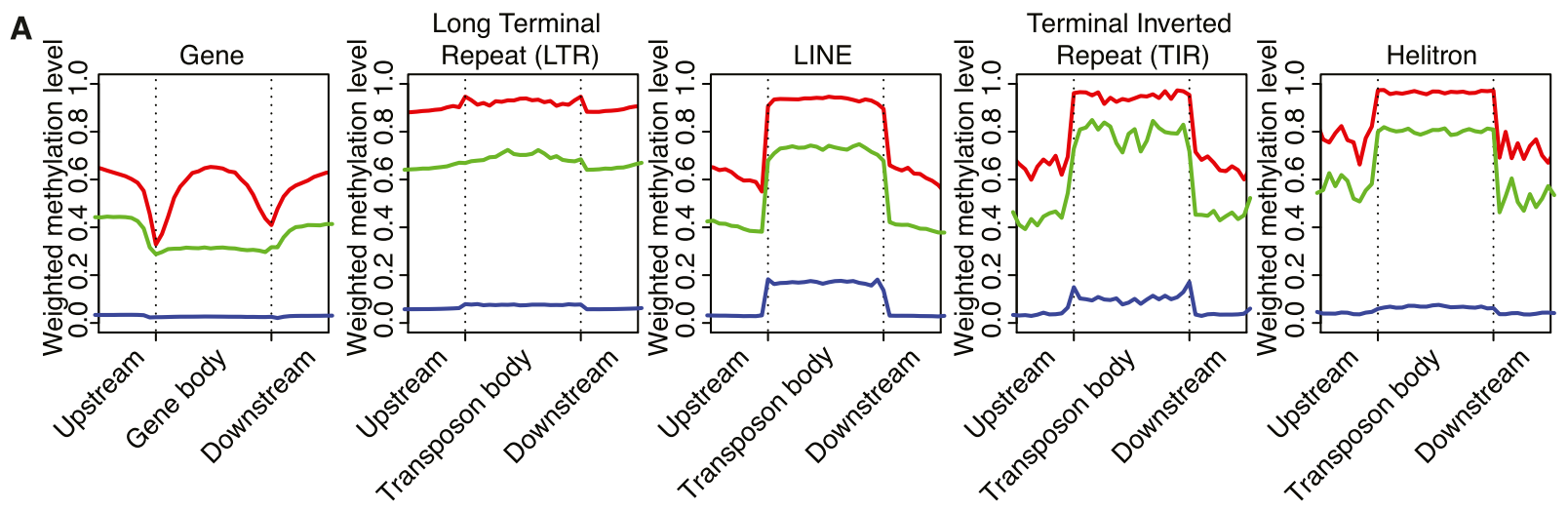

$-\mathrm{mCG}-\mathrm{mCHG}-\mathrm{mCHH}$

B

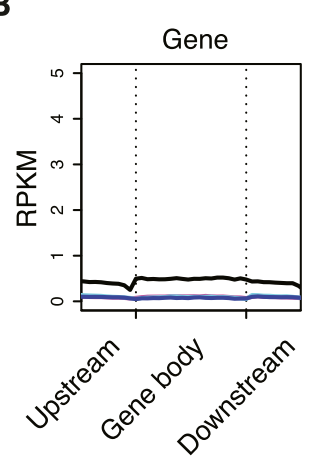

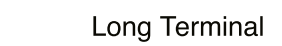

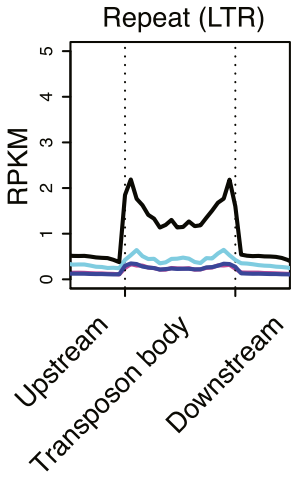

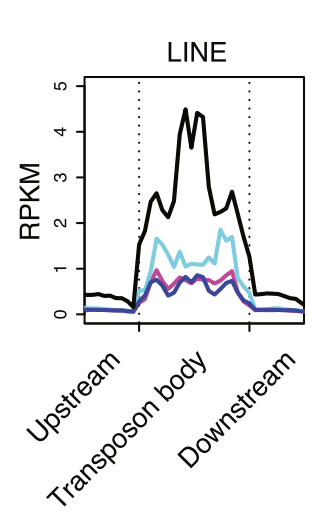
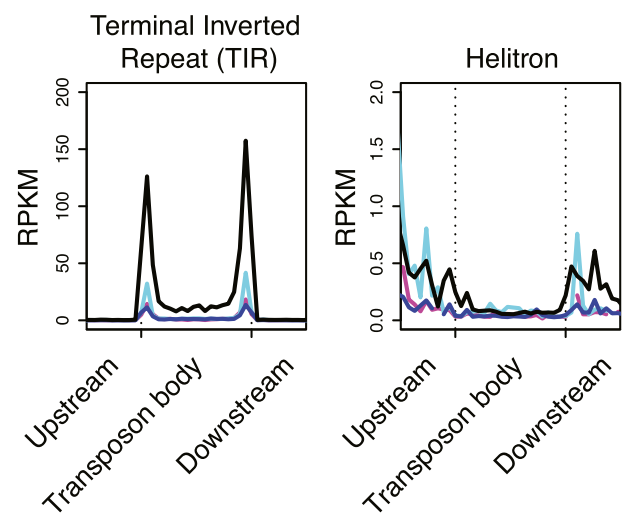

C
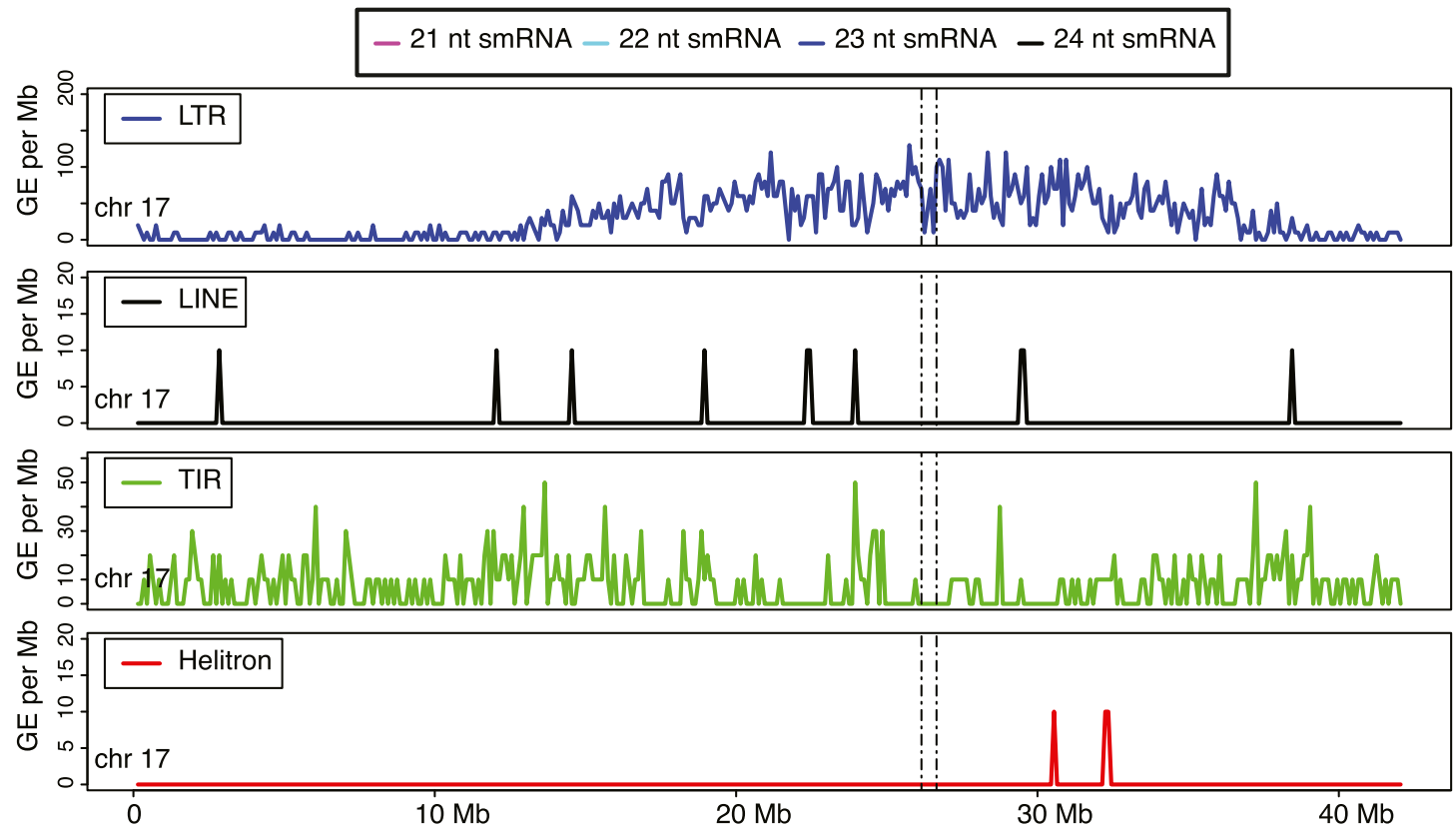

Figure 2. Characterization of gene body and transposon DNA methylation in soybean. ( $A$ ) The distribution of $\mathrm{mCG}$, $\mathrm{mCHG}$, and $\mathrm{mCHH}$ densities and $(B)$ the distribution of 21-24 nt smRNA levels in gene bodies, Long terminal repeats (LTRs) retrotransposons, LINE retrotransposons, terminal inverted repeat (TIR) DNA transposons, and Helitron DNA transposons including $\pm 4 \mathrm{~kb}$ from the start and stop codons. (C) Chromosome-wide density of LTR, LINE, TIR, and Helitron transposons. Only data points between $1 \%$ and $99 \%$ quintiles were used to generate values for each bin in the plots presented in $A$ and $B$. 
A

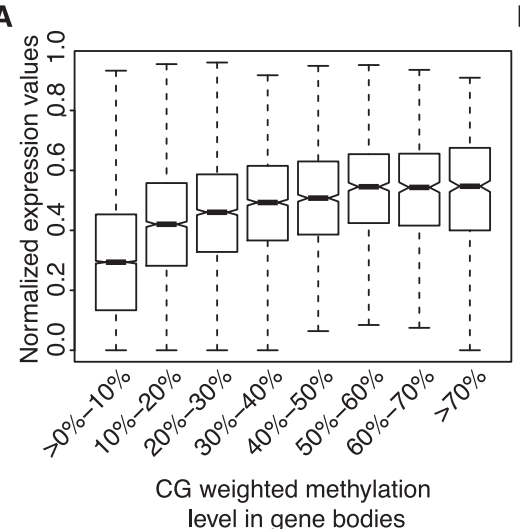

D

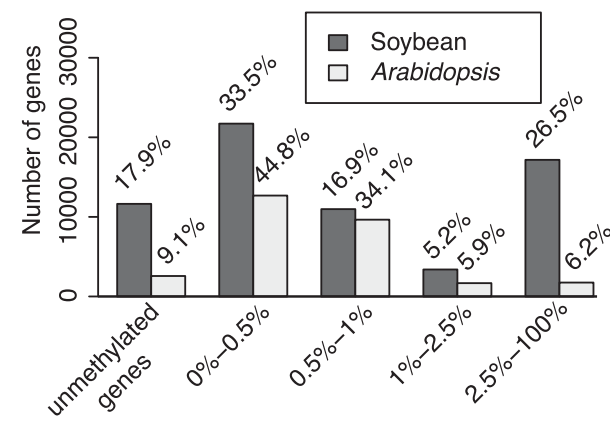

CHG weighted methylation level in exons

$\mathbf{F}$
B

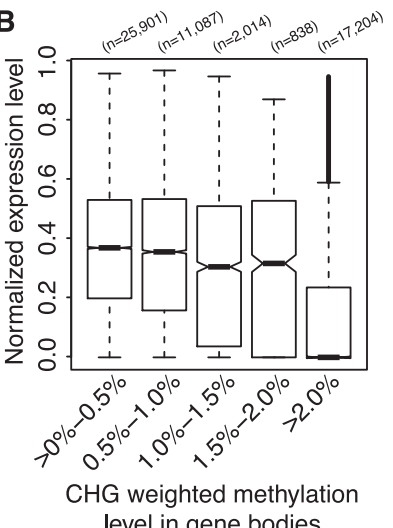

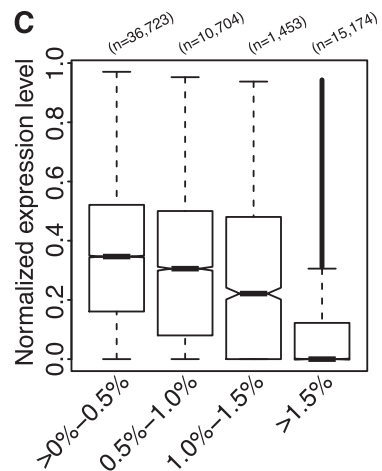

$\mathrm{CHH}$ weighted methylation level in gene bodies

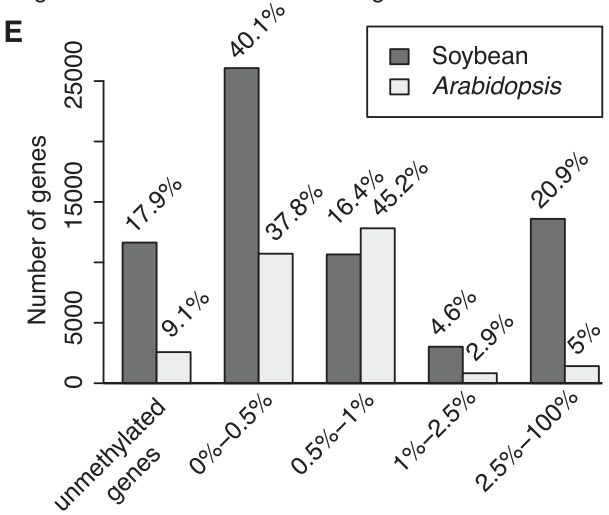

$\mathrm{CHH}$ weighted methylation level in exons

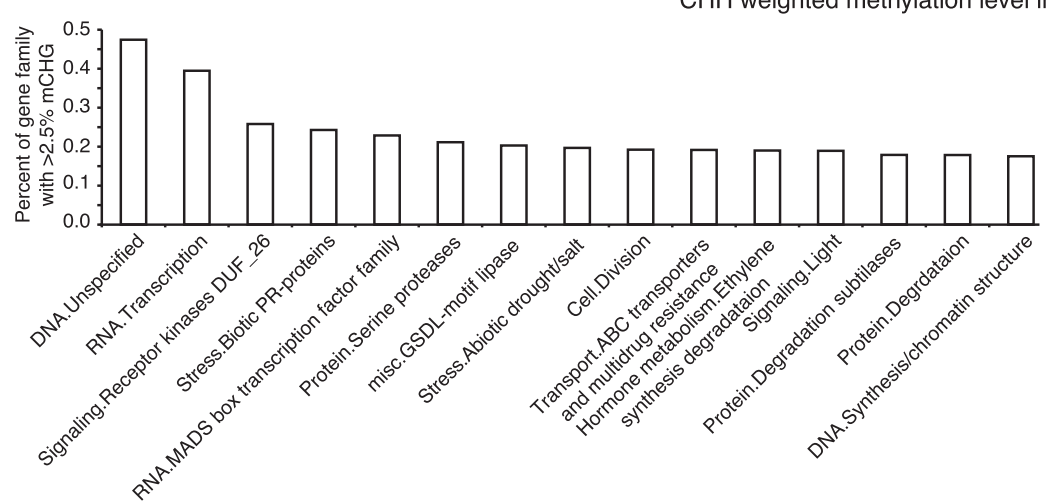

Figure 3. The association between DNA methylation and gene expression levels in soybean. $(A)$ Increasing levels of CG gene-body methylation is correlated with increasing levels of gene expression. Box plot representation of different levels of CG gene-body methylation is displayed along the $x$-axis, whereas normalized gene expression levels are plotted on the $y$-axis. Genes containing $>0.5 \%$ non-GC methylation were filtered from this analysis. Increasing methylation levels of both $\mathrm{CHG}(B)$ and $\mathrm{CHH}(C)$ sites are associated with decreasing levels of gene expression levels. $(D, E)$ The soybean genome contains a higher proportion of RdDM-targeted loci compared to the Arabidopsis thaliana genome. Fraction of genes ( $y$-axis) targeted by varying levels of $\mathrm{CHG}(D)$ and $\mathrm{CHH}(E)$ DNA methylation ( $x$-axis). Unmethylated genes were defined as loci containing $<0.5 \% \mathrm{CG}$, $\mathrm{CHG}$, and $\mathrm{CHH}$ methylation. $(F)$ Gene families containing the highest fraction of members containing $>2.5 \%$ weighted $\mathrm{CHG}$ methylation. Only gene families with more than 100 members were considered in this analysis and only the top fifteen classes are displayed.

which is consistent with the ability of the RdDM pathway to actively repress subsets of soybean genes.

The soybean genome contains $\sim 66,000$ protein-coding genes (Schmutz et al. 2010) in contrast to the $\sim 27,000$ protein-coding genes present in the Arabidopsis thaliana Col-0 genome (The Arabidopsis Genome Initiative 2000), which reflects recent whole genome duplications (Schmutz et al. 2010). The soybean LD genome contains higher amounts of all three types of DNA methylation (Fig. 1A) and greater proportions of CHG methylation when compared to Arabidopsis thaliana (Fig. 1B), which is likely a result of the RdDM pathway more actively targeting genes in soybean (Fig. $3 \mathrm{D}, \mathrm{E})$. We defined genes as possible targets of RdDM that contained $>2.5 \%$ of either $\mathrm{CHG}$ or $\mathrm{CHH}$ weighted methylation levels as these levels had a measurable effect on gene expression. Although $\sim 6 \%$ and $\sim 5 \%$ of the Arabidopsis thaliana genes are targeted, respectively, by CHG and $\mathrm{CHH}$ methylation, $\sim 26 \%$ and $\sim 20 \%$ are targeted in soybean, representing an approximately fourfold increase (Fig. 3D). A closer inspection of the top 15 classes of genes that are most frequently targeted by $\mathrm{CHG}$ and $\mathrm{CHH}$ methylation (Fig. 3F) revealed enrichment for biotic pathogen re- 
sponse proteins, MADS-box transcription factors and protein degradation machinery, which is similar to the three gene families most targeted by CHG and $\mathrm{CHH}$ methylation in Arabidopsis thaliana (Schmitz et al. 2013).

\section{RdDM targets recently duplicated paralogs}

Recently duplicated genes in Arabidopsis thaliana show a strong preference for maintenance of methylation states (Widman et al. 2009), but analysis of the effects of DNA methylation on gene expression in this species is limited by a relatively low number of paralogs. In contrast, the whole-genome duplications in soybean have resulted in almost 10,000 identified pairs of paralogs, and it is plausible that these recent duplications underlie the increased number of genes targeted by $\mathrm{CHG}$ and $\mathrm{CHH}$ methylation detected above. To determine if paralogs are more likely to contain one paralog that is enriched for non-CG methylation, the $\mathrm{CG}, \mathrm{CHG}$, and $\mathrm{CHH}$ methylation levels of these pairs were plotted against one another (Fig. 4A-C). For CG methylation, the density plot revealed that most pairs are methylated at relatively equal levels, as the diagonal of the plot is most dense (Fig. 4A). This pattern reflects that CG gene-body methylation, which is not repressive in nature, is largely maintained between paralogs similar to previous reports for orthologs (Takuno and Gaut 2013). This pattern contrasts with $\mathrm{CHG}$ and $\mathrm{CHH}$ methylation in which the vast majority of paralogs are unmethylated or methylated at very low levels and present near the lower left corner of the plot (Fig. 4B,C). Interestingly, clear examples of differentially methylated paralogs are present along the zero plane of the $x$ - or $y$-axis (Fig. 4B-F; Supplemental Table 3). A total of $602 / 9793$ paralogs in soybean were differentially targeted by non-CG methylation, which represents a significant enrichment $\left(P\right.$-value $\left.<6.648 \times 10^{-7}\right)$ (Methods) when compared to Arabidopsis thaliana paralogs (4/497). Furthermore, the methylated forms of the paralogs were expressed at significantly lower levels ( $P$-value $<2 \times 10^{-16}$, Wilcoxon signed-rank test) and resided closer to transposons when compared to their unmethylated counterparts (Supplemental Fig. 7). In fact, 56/602 differentially methylated paralogs were strictly defined by the presence of a transposon that was targeted by $\mathrm{CG}, \mathrm{CHG}$, and $\mathrm{CHH}$ methylation overlapping the gene space. These results indicate that one potential route to gene expression variation in recently duplicated genomes among paralogs is through the actions of DNA methylation and in some cases nearby transposon sequences.

\section{Variation in DNA methylation among soybean parental and recombinant inbred lines}

To explore the potential for natural variation of DNA methylation patterns in soybean, MethylC-seq and RNA-seq were performed on the LDX01-1-165 germplasm (hereafter referred to as "LDX"). Additionally, the methylomes and transcriptomes of two recombinant
B

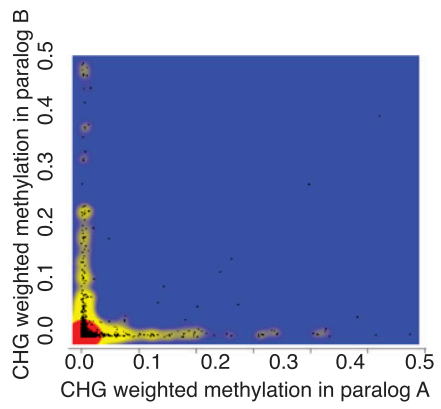

D
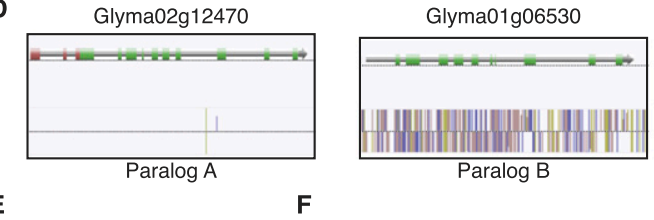

Glyma06g11210 Glyma04g12090 Glyma06g21000 Glyma04g33280
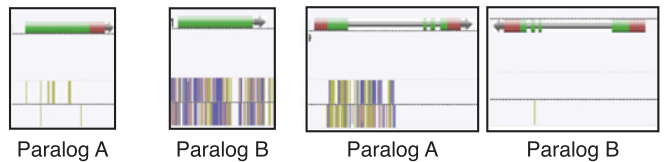

Paralog B

Paralog $\mathrm{B}$

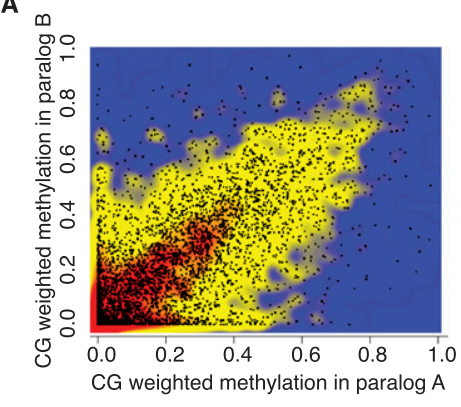

(1)

Figure 4. Pairwise methylation levels for paralog gene pairs. (A-C) Pairwise plots of $\mathrm{CG}(A), \mathrm{CHG}(B)$, ylation between paralog pairs in the LD methylome. (Gold lines) mCG; (purple lines) mCHG; (pink lines) $\mathrm{mCHH}$. Loci containing $>0.5 \%$ non-GC methylation levels were excluded from the plot in $A$, and genes containing $>2.5 \% \mathrm{mCHG}$ in $B$ and $C$ were considered targets of RdDM.

inbred lines (RILs) that were derived from the LD and LDX parental germplasms (Kim et al. 2011) were profiled to enable determination of the heritability of DNA methylation states upon combination of newly introduced genetic variants. Over 100 million 101-bp aligned reads were recovered for LDX and the two RILs (R-11268 and R-11272) representing greater than $\sim 11 \times$ coverage for each sample (Supplemental Table 1). RNA-seq data was acquired for all three lines in biological triplicates and had at least 30 million aligned reads per sample (Supplemental Table 2).

The single-base resolution bisulfite-sequencing data enabled identification of single methylation polymorphisms (SMPs) (Schmitz et al. 2011), differentially methylated regions only in the CG context (CG-DMRs), and differentially methylated regions in all types of DNA methylation (C-DMRs) present between the four sequenced lines. In total, 280,712 CG-SMPs, 703,685 CHG-SMPs, and 9,819,894 CHH-SMPs were identified between the parental and RIL methylomes. CG-SMPs were more abundant in genes and more specifically in introns compared to transposon and intergenic sequences (Supplemental Fig. 8A), whereas CHG- and CHHSMPs were more abundant in transposon sequences (Supplemental Fig. 8A). The patterns of CG-SMP variability are similar to the patterns observed for CG-DMRs (Supplemental Fig. 8B).

A total of 3241 CG-DMRs were identified among the four lines sequenced, and $61 \%$ of these overlapped gene bodies and were found in similar distributions across gene bodies (Supplemental Fig. 8C), similar to the patterns of CG gene-body methylation (Fig. 2A). To determine the potential impact of these CG-DMRs on gene expression, the methylation levels of each CG-DMR were plotted against the gene expression level of the locus overlapping the position of each CG-DMR within each sample (Supplemental Fig. 8D). Regardless of the methylation level present within each of the 
CG-DMRs, the gene expression levels remained constant, indicating that in these limited samples no clear correlation between CG-DMR methylation levels and gene expression levels was detected (Supplemental Fig. 8D). Although CG-DMRs are an average size of $431 \mathrm{bp}$ and preferentially found in gene bodies, C-DMRs, of which there are 1416 , are an average size of $1162 \mathrm{bp}$, also abundant in gene bodies (Supplemental Fig. 8E,F), and are most abundant within $1 \mathrm{~kb}$ of the transcriptional start site of genes (Supplemental Fig. 8G). However, for C-DMRs that overlapped genes, increasing levels of methylation within each C-DMR are correlated with decreasing levels of gene expression (Supplemental Fig. 8H), indicating that these types of DMRs can contribute to the variation in gene expression observed between different genotypes.

Additionally, whole-genome sequencing data were obtained for the LD and LDX parental lines and SNPs were identified using the SHORE analysis pipeline (Ossowski et al. 2008). As expected, most of the SNPs identified in each genotype sequenced were located in intergenic regions, but significant fractions were identified in protein-coding genes (Supplemental Table 4). Major effect mutations were identified and defined as SNPs that abolished known start and stop codons, as well those SNPs that created premature stop codons. We hypothesized that loci that are targeted by non-CG methylation may accumulate major effect mutations at a higher rate than nonunmethylated loci because they are not frequently expressed in sporophytic tissues, but were unable to find any significant correlation to support this claim $\left(\chi^{2}\right.$ test, $P$-value $\left.=0.93\right)$. Therefore, it is likely that the repressive DNA methylation at these loci has evolved for other purposes, some of which may be important for plant development (Zemach et al. 2010a; Martínez and Slotkin 2012; Schmitz et al. 2013), germ line maintenance (Slotkin et al. 2009; Calarco et al. 2012; Ibarra et al. 2012) and/or responses to biotic stresses (Dowen et al. 2012).

\section{Cosegregation analysis of DMRs and genotype}

Although there is extensive methylation variation within and between plant species, the heritability of methylation variants has not been extensively explored in a population on a genome-wide scale. To understand the stability and heritability of methylation variants, we examined the methylation levels of CG- and C-DMRs in homozygous regions of R-11268 and R-11272 and compared them to their parental states in LD and LDX (Fig. 5A; Methods). In total, 3670/ 4474 and 1924/2048 of the methylation levels of CG-DMRs and CDMRs, respectively, in R-11268 and R-11272 cosegregated with the parental state (Supplemental Tables 5, 6), whereas 254/4981 for CGDMRs and 122/2048 for C-DMRs were found to contain the methylation state of the other parent. This would suggest that methylation states of some DMRs are due to distant loci or are epigenetically unstable, as has been observed in Arabidopsis thaliana and maize (Becker et al. 2011; Eichten et al. 2011; Schmitz et al. 2011), although other possible explanations could include incorrect assignment of DMRs to their genotype and low sequencing coverage of DMRs.

\section{Population-wide identification of methylQTL}

The epigenomics approach undertaken in this study enabled identification of methylation variants that are both linked and unlinked to genotype (although the latter is much rarer), but the low sample size of only two RILs makes understanding the population dynamics of methylation states difficult. However, because the methylation status of the majority of C-DMRs cosegregated with their genotype in the two RILs, it should be possible to map potential causal variants for the methylation variation in this population. DNA methylome data were acquired for an additional 81 lines from the RIL population and QTL mapping for each CDMR was performed, which revealed evidence for a methylQTL for 1293/1416 (91\%) C-DMRs (Fig. 6A; Supplemental Table 7). Of the identified methylQTL, 1260/1293 mapped locally to the C-DMR (Fig. 6B,C; Supplemental Fig. 9), whereas 33 mapped to a different chromosome from where the C-DMR was located (Fig. 6D,E). Lastly, heritability estimates for each methylQTL were calculated, which revealed that many methylQTL could explain a large proportion of the methylation variation of their associated C-DMR (Fig. 6F). The methylQTL with lower heritability estimates could be reflective of methylation variants that display higher epimutation rates, possibly because these variants are not directly linked to a genetic variant. Future efforts to identify causal genetic variants will be necessary to understand the stability of different classes of methylation variants.

\section{Discussion}

Studies in plants have led to major advances in the field of epigenetics, especially with regard to natural epigenetic variation (Weigel and Colot 2012). Plant genomes contain cytosine DNA methylation that occurs not only in the CG context but also in CHG and $\mathrm{CHH}$ contexts (Cokus et al. 2008; Lister et al. 2008), and these specific signatures are often indicative of the type of regulation occurring at the methylated locus. Epigenomic techniques have revealed widespread natural variation in DNA methylation in a range of plant species (Vaughn et al. 2007; Zhang et al. 2008; He et al. 2010; Becker 
A

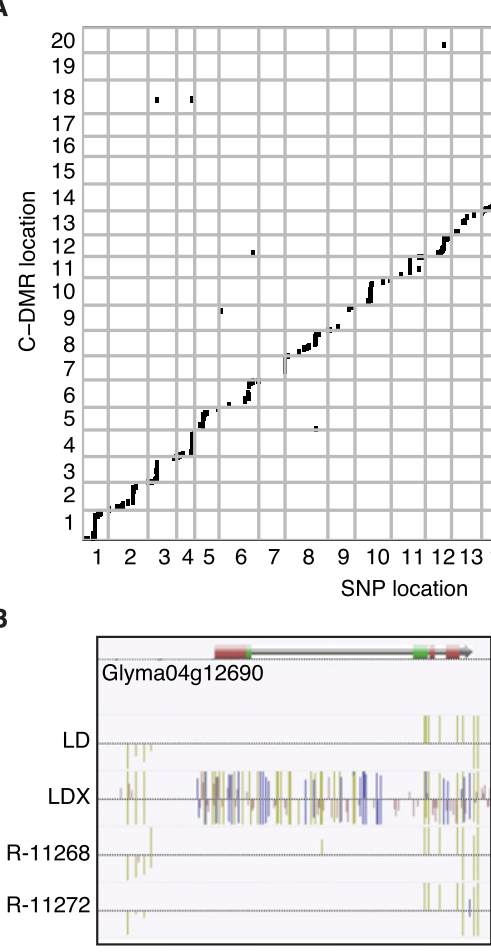

C

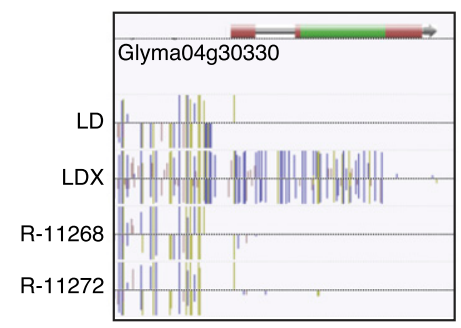

$F$

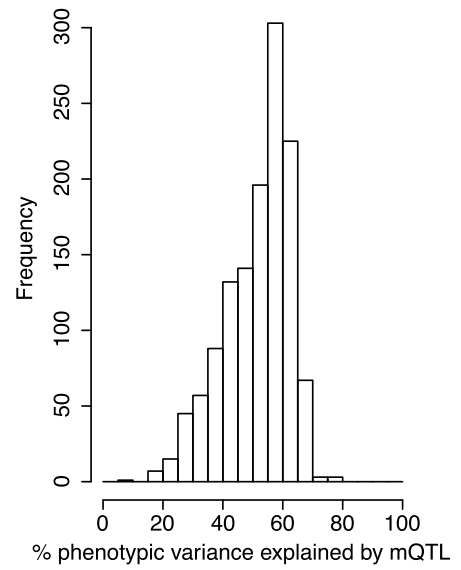

D

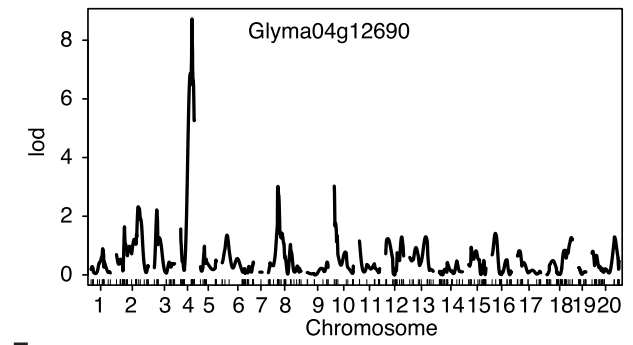

E

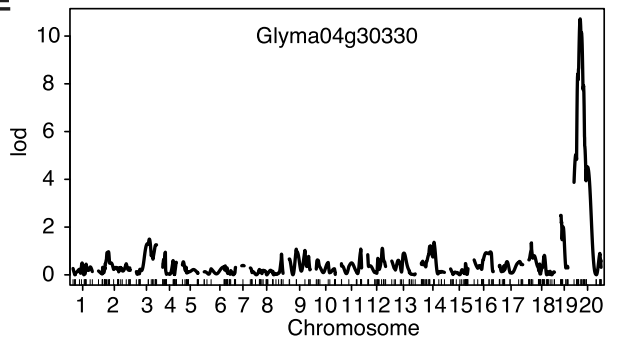

Figure 6. Population-level analysis of methylation variants and identification of methylQTL. ( $A$ ) Scatter plot of the location (x-axis) of all single methylQTL identified for C-DMRs ( $y$-axis). $(B, C)$ DNA methylation profiles for LD, LDX, R-11268 and R-11272 of two different C-DMRs. (Gold lines) mCG; (purple lines) $\mathrm{mCHG}$; (pink lines) $\mathrm{mCHH}$. (D) An example QTL map of a local methylQTL and $(E$ ) a distant methylQTL. (F) Broad-sense heritability estimates for all single methylQTL/C-DMR pairs.

et al. 2011; Eichten et al. 2011; Groszmann et al. 2011; Schmitz et al. 2011; Greaves et al. 2012; Shen et al. 2012), but we are only beginning to understand the role of DNA methylation and modes of inheritance for different methylation variants.

Although whole-genome bisulfite sequencing data revealed natural epigenomic variation between soybean germplasms, it revealed a greater proportion of the methylome that was invariably methylated. In fact, although the soybean genome is approximately eightfold larger than the Arabidopsis thaliana genome, it contained proportionally more DNA methylation, which was disproportionally present in $\mathrm{CHG}$ and $\mathrm{CHH}$ sites, indicating that $\mathrm{RdDM}$ is more active in the soybean genome. A closer inspection of the regions of the genome targeted by non-CG methylation revealed that approximately fourfold more protein-coding regions are actively silenced. Given the recent genome duplications present in the soybean genome (Schmutz et al. 2010), this additional targeting could indicate that these genes are being purged from the genome or expressed at very low levels until subfunctionalization occurs (Roulin et al. 2012). If this were the case, it would be expected that these loci accumulate higher frequencies of major effect mutations compared to loci that do not contain non-CG methylation, but we were unable to find any evidence to support this possibility. Therefore, these genes might be present within the genome in a transcriptionally inert state, which could allow them to function during situations that result in global reactivation of loci that contain non-CG methylation, which can occur upon biotic infection or during certain developmental stages (Zemach et al. 2010a; Dowen et al. 2012; Martínez and Slotkin 2012; Schmitz et al. 2013; Yu et al. 2013) similar to reports for transposons (Ohtsu et al. 2007; Slotkin et al. 2009; Li et al. 2010; Calarco et al. 2012; Ibarra et al. 2012).

One additional role of non-GC methylation in soybean genome found in this study is differential targeting of paralogs, which drives gene expression variation. Whether or not this is restricted to soybean or widespread among additional crop genomes is unknown. Although, it was recently reported that differential accumulation of H3K27me3 between maize paralogs preferentially occurs in recently duplicated regions of the genome (Makarevitch et al. 2013). Therefore, it is likely that there are multiple epigenomic mechanisms that can lead to expression variation and will undoubtedly be an interesting topic for future investigations.

In this study, we have revealed that the majority of C-DMR methylation variants identified cosegregated with the genetic background from which they were derived, but there were rare examples of uncoupling between methylation states and genotype, which potentially provide an additional source for natural epigenetic variation. One possible mechanism to explain the methylation variants that did not cosegregate with their genotype could include paramutation, as has been observed in maize (Patterson et al. 1993; Arteaga-Vazquez and Chandler 2010), but analysis of these C-DMRs did not reveal such events. For the C-DMR methylation variants that do follow standard laws of inheritance, their stability is likely a result of being targeted by non-GC methylation, a process that would enact a double-hit mechanism by taking advantage of the activities of maintenance methyltransferases at CG and CHG sites (Ronemus et al. 1996; Mathieu et al. 2007; Du et al. 2012) in addition to small RNA directed methylation by de novo methyltransferases at all cytosines (Cao et al. 2003; Teixeira et al. 2009).

The heritability of methylation states of C-DMRs suggested that some of these C-DMRs might have arisen as a consequence of genetic variants, as has been observed with the PAI gene family and the AtFOLT1 paralogs in Arabidopsis thaliana (Bender and Fink 1995; Durand et al. 2012), whereas others could have arisen and segregated independently of genetic variants like QQS (Silveira et al. 2013). In fact, QTL mapping of this population uncovered a

\section{Genome Research www.genome.org}


methylQTL for $\sim 91 \%$ of the C-DMRs, indicating that these C-DMRs are either linked to a genetic variant or are stably inherited, whereas the methylation states of the remaining 9\% of C-DMRs likely do not follow standard laws of inheritance. The vast majority of these methylQTL mapped to the C-DMR, but there was also clear evidence for methylQTL located on different chromosomes than the C-DMR. It should be noted that genome rearrangements in the parental lines or misassemblies in the reference genome could explain some of these distant methylQTL. For example, there are four C-DMRs on chromosome 18 that are all significantly associated with a single marker on chromosome 4 . In any case, these methyQTL are candidate regions that in many cases likely harbor a causal genetic variant(s) underlying the methylation variation of the respective C-DMR. Identifying the types of causal variants that lead to methylation variation in plants will require large-scale epigenomic projects using natural plant populations, which will enable higher-resolution association mapping. In fact, a number of phenotyping and sequencing projects are already underway that will advance the use of quantitative genetic approaches to understanding natural variation of morphological or molecular phenotypes of interest (Lam et al. 2010; Cao et al. 2011; Gan et al. 2011; Huang et al. 2012).

\section{Methods}

\section{Plant material}

The two parental lines, LD00-2817P (Diers et al. 2010) and LDX011-65 (Brucker et al. 2005), were used to create the studied RIL population (see Supplemental Methods for additional information).

\section{Construction of sequencing libraries}

DNA sequencing libraries for LD and LDX were constructed as reported in Johnson et al. (2012). MethylC-seq libraries were constructed according to Schmitz et al. (2011). RNA-seq libraries were constructed using the Illumina TruSeq Kit v2 according to the manufacturer's guidelines.

\section{Sequencing}

gDNA-seq, MethylC-seq, and RNA-seq libraries were sequenced using an Illumina HiSeq 2000 according to the manufacturer's instructions. gDNA-seq and MethylC-seq libraries were sequenced for 101 cycles, and RNA-seq libraries were sequenced for 51 cycles. An additional run of paired-end $2 \times 101$ bp sequencing was performed for the gDNA libraries.

\section{RNA-seq analysis}

All RNA samples were performed as biological triplicates for each genotype. Illumina HiSeq2000 output files in the FASTQ format were aligned to the Glycine max reference genome version 1.0 (Schmutz et al. 2010) (Gm1.0 ftp://ftp.jgi-psf.org/pub/ compgen/phytozome/v8.0/Gmax_v1.0/) using Bowtie version 0.12.7 (Langmead et al. 2009) and TopHat version 1.3.3 (Trapnell et al. 2009) (flags $=-$ g 1, -F 0). Gene expression values were calculated using Cufflinks version 1.1.0 (flags $=-\mathrm{F} 0,-\mathrm{b},-\mathrm{N}$ ) (Trapnell et al. 2010).

\section{MethylC-seq analysis}

MethylC-seq analysis was performed similarly to Lister et al. (2011) with some modifications (see Supplemental Methods).

\section{Identification of DMRs}

To identify DMRs, a root mean square test (Perkins et al. 2011) was applied to all cytosines, which required building a contingency table where the rows indicated a particular sample and the columns indicated the number of reads that supported a methylated cytosine or an unmethylated cytosine at each position in a given sample. Using 10,000 permutations, the $P$-values were simulated; and for each new permutation, a contingency table was generated by randomly assigning reads to cells with a probability equal to the product of the row marginal and column marginal divided by the total number of reads squared. To increase the efficiency of this process, if a $P$-value returned 100 permutations with a statistic greater than or equal to the original test statistic, permutations were discontinued (i.e., we used adaptive permutation testing). To determine a $P$-value cutoff that would control the false discovery rate (FDR) at a rate of 1\%, the procedure in Bancroft et al. (2013) was applied. Briefly, this method first generates a histogram of the $P$-values and calculates the expected number of $P$-values to fall in a particular bin under the null. This expected count is computed by multiplying the width of the bin by the current estimate for the number of true null hypotheses $\left(m_{0}\right)$, which is initialized to the number of tests performed. It then looks for the first bin (starting from the most significant bin and working its way toward the least significant) where the expected number of $P$-values is greater than or equal to the observed value. The differences between the expected and observed counts in all the bins up to this point are summed, and a new estimate of $m_{0}$ is generated by subtracting this sum from the current total number of tests. This procedure was iterated until convergence, which we defined as a change in the $m_{0}$ estimate less than or equal to 0.01 . With this $m_{0}$ estimate, we were able to estimate the FDR of a given $P$-value by multiplying the $P$-value by the $m_{0}$ estimate (the expected number of positives at that cutoff under the null hypothesis) and dividing that product by the total number of significant tests we detected at that $P$-value cutoff. We chose the largest $P$-value cutoff that still satisfied a 1\% FDR requirement. Once this $P$-value cutoff was chosen, significant sites were combined into blocks if they were within 500 bases of one another and had methylation changes in the same direction (e.g., sample A was hypermethylated and sample B was hypomethylated at both sites). Three different types of DMRs were identified from the data set-C-DMR (a change in all three contexts), CG-DMR (a change only in the CG context), and CH-DMR (a change in either the CHG or the CHH contexts). Furthermore, C-DMR, CG-DMR, and CH-DMR blocks that contained fewer than 10, 5, and 5 differentially methylated sites were discarded, respectively. Final lists of C-DMRs required an overlap with both a CG-DMR and a CH-DMR, and the final list of CG-DMRs were only retained if they did not overlap a C-DMR or a CH-DMR.

\section{Weighted methylation levels}

Weighted methylation levels were computed as described in Schultz et al. (2012).

\section{Identification of "early" and "recent" whole genome duplications (WGD) and paralogs}

Synteny blocks were identified with DAGchainer (Haas et al. 2004), based on anchor points determined using the NCBI blastp program $\left(E\right.$-value $\left.\leq 1 \times 10^{-10}\right)$, filtered to the top reciprocal best matches per chromosome pair. Synteny blocks from Glycine ("recent") WGD were identified as those with median $K_{\mathrm{s}}$ values $\leq 0.35$ per block, and blocks from the legume ("old") WGD were identified as those with median $K_{\mathrm{s}}$ values $>0.35$ and $\leq 1.5$ per block. $K_{\mathrm{s}}$ values per gene were determined using the codeml program from 
the PAML package, version 4.4 (Yang 2007). The paralogs used in this study and the descriptions of how they were identified were obtained from Supplemental Table 4 in a previously published study (Libault et al. 2010). Furthermore, our analysis on soybean paralogs was strictly focused on genes harboring two copies in the genome that were strictly duplicated genes from the recent WGD. Differentially methylated paralogs were identified by searching for pairs in which one paralog contained $>2.5 \% \mathrm{CHG}$ methylation and the other paralog had $<0.5 \%$ CHG methylation. Arabidopsis thaliana paralogs were obtained from Supplementary Material in a previously published study (Ganko et al. 2007). The "prop.test" function in $\mathrm{R}$ was used to estimate if the proportion of significantly differentially methylated paralogs in soybean was greater than in Arabidopsis thaliana.

\section{Small RNA analysis}

smRNA data were downloaded from the National Center for Biotechnology for Information SRA012752 (Tuteja et al. 2009). These small RNAs were isolated from young cotyledons from the Williams accession. Raw smRNA data were preprocessed by removing the $3^{\prime}$ adapter sequence and any sequencing reads under $16 \mathrm{bp}$. Reads passing these filters were aligned to the Gm1.0 reference genome using the Bowtie (v0.12.7) and the following parameters: -e 1 -1 20 -n 0 -a -m 1000-best-nomaqround. Only reads that contained perfect matches within the genome and that did not have more than a thousand locations were retained for further data analysis.

\section{Identification of SNPs}

SNPs were identified using the SHORE variant identification software package (Ossowski et al. 2008) using the BWA aligner (Li and Durbin 2009), allowing up to 5\% errors per read and a max of three gaps. Any SNP with a quality score of 25 or above was used for further analysis.

\section{SNP effects}

The impact of SNPs on coding regions were determined using the SnpEff tool ("SnpEff: Variant effect prediction"; http://snpeff. sourceforge.net) (Cingolani et al. 2012) using a Glycine max reference file.

\section{Genetic reconstruction of RILs based on bisulfite sequencing reads}

Only SNPs that distinguished the LD and LDX parental lines were used to determine the genotypes of the RILs. All SNP pairs containing C-T, T-C, A-G, or G-A changes were excluded because we were unable to distinguish those SNPs due to bisulfite conversion of reads. Next, the number of reads in each RIL that matched the LD or LDX alleles was determined using the bisulfite converted reads, and any position containing at least four reads matching a parent was considered for further analysis. A position was determined heterozygous if at least four reads were identified that supported each parent. Using these data, a score was assigned as 1.0 for the LD genotype, 0 for heterozygous positions, and -1.0 for the LDX genotype. Next, the genome was divided into 100-kb bins, and the score for each bin was computed by averaging the scores of each position within it. Only bins with greater than 10 SNPs were included in the calculation. Next, we assigned tags to bins based on the score. Bins with a score greater than 0.5, were tagged as LD, whereas bins with a score of less than - 0.5 were assigned as LDX. Bins without a score were kept untagged, and the rest (with score between -0.5 and 0.5 ) were labeled as heterozygous. Lastly, large regions were formed by concatenating adjacent bins with the same tag (LD or LDX) or bins that had the same tag but were spaced by untagged bins.

\section{Assignment of DMRs to genotype}

DMRs were assigned to genotypes based on their overlap with the genetic reconstruction of the R-11268 and R-11272 RILs. If a DMR was within one reconstructed region, then its genotype was the same as that region. To determine the parental methylation state of each DMR, the weighted methylation level was computed for each genotype (LD, LDX, R-11268, and R-11272). If the value of RIL was within $20 \%$ of either one of the parents, then the DMR was assigned to that parent. If the value of RIL was more extreme or between either parent-weighted methylation level, then it was labeled "No association detected." To compare the methylation level of DMRs in the offspring and the parents, we computed the ratio of the weighted methylation level of each region in the RILs and then subtracted the lower weighed methylation level from each parent to determine the absolute difference between the weighted methylation levels of two parents. The ratio can be represented using the following equation:

$$
\begin{aligned}
\text { Ratio }= & \text { Met }-\min \left(\operatorname{Met}_{L D}, \text { Met }_{L D X}\right) \max \operatorname{Met}_{L D}, \text { Met }_{L D X} \\
& -\min \left(\text { Met }_{L D}, \text { Met }_{L D X}\right)
\end{aligned}
$$

where Met is the weighted methylation level of this region in a RIL, and $\mathrm{Met}_{L D}$ and $\mathrm{Met}_{L D X}$ are the weighted methylation levels in LD and LDX, respectively.

\section{QTL mapping of C-DMRs}

The R/qtl package (Broman et al. 2003) was used to map QTL for each C-DMR. First, missing genotypes were imputed using the "fill.geno" function. Next, genotypes between SNP markers were simulated and imputed using the "sim.geno" function with the following parameters: "step $=1$, error.prob $=0.01$, n.draws $=20$." Then, for each C-DMR, the "scanone" function (option: "model='np' ") was used to compute a LOD score for each SNP marker across the genome. Permutation testing (1000 times) was used to estimate the significance of each LOD peak(s). methylQTL was defined as the closest significant SNP marker $(P$-value $<0.01)$ to the summit of the highest peak. Only the single highest LOD score was reported for each C-DMR. The broad-sense heritability of each QTL was estimated by doing an ANOVA analysis using the "fitqtl" function.

\section{Additional analyses}

For analysis of SMPs, transposons, and for information regarding gene annotations Arabidopsis thaliana data used in this study, see Supplemental Methods.

\section{Data access}

The data generated for this work have been deposited in the NCBI Gene Expression Omnibus (GEO; http://www.ncbi.nlm.nih.gov/ geo/) and are accessible through accession number GSE41753. Genome sequencing data have been deposited in the NCBI Sequence Read Archive (SRA; http://www.ncbi.nlm.nih.gov/sra/) under accession number SRA060034. Processed data can be visualized at http:// neomorph.salk.edu/soybean_RIL_methylomes/browser.html.

\section{Acknowledgments}

We thank Mathew Lewsey and Matthew Schultz for their comments on this manuscript; Steve Cannon for identification of the

\section{Genome Research www.genome.org}


"early" and "recent" whole genome duplications; and Justin Borevitz and Riyan Cheng for discussions about QTL analysis. R.J.S. was supported by a National Institutes of Health postdoctoral fellowship (K99GM100000). This research was funded by the Howard Hughes Medical Institute and the Gordon and Betty Moore Foundation (through Grant GBMF3034); the Mary K. Chapman Foundation; and the National Science Foundation (MCB-0929402 and MCB1122246) to J.R.E. This work was also supported by the Division of Chemical Sciences, Geosciences, and Biosciences, Office of Basic Energy Sciences of the U.S. Department of Energy through Grant DE-FG02-08ER15309, as well as funding from the United Soybean Board and North Central Soybean Research Program to G.S.

Author contributions: R.J.S., J.R.E., and G.S. conceived and designed experiments. R.J.S., O.V.L., M.A.U., and J.R.N. performed experiments. R.J.S., Y.H., S.M.K., T.J., and B.D. analyzed the data. D.X. provided guidance and valuable insight. R.J.S. wrote the paper.

\section{References}

The Arabidopsis Genome Initiative. 2000. Analysis of the genome sequence of the flowering plant Arabidopsis thaliana. Nature 408: 796-815.

Arteaga-Vazquez MA, Chandler VL. 2010. Paramutation in maize: RNA mediated trans-generational gene silencing. Curr Opin Genet Dev 20: 156-163.

Bancroft T, Du C, Nettleton D. 2013. Estimation of false discovery rate using sequential permutation p-values. Biometrics 69: 1-7.

Becker C, Hagmann J, Müller J, Koenig D, Stegle O, Borgwardt K, Weigel D. 2011. Spontaneous epigenetic variation in the Arabidopsis thaliana methylome. Nature 480: 245-249.

Bender J, Fink GR. 1995. Epigenetic control of an endogenous gene family is revealed by a novel blue fluorescent mutant of Arabidopsis. Cell 83: 725734.

Broman KW, Wu H, Sen S, Churchill GA. 2003. R/qtl: QTL mapping in experimental crosses. Bioinformatics 19: 889-890.

Brucker E, Carlson S, Wright E, Niblack T, Diers B. 2005. Rhg1 alleles from soybean PI 437654 and PI 88788 respond differentially to isolates of Heterodera glycines in the greenhouse. Theor Appl Genet 111: 44-49.

Calarco JP, Borges F, Donoghue MT, Van Ex F, Jullien PE, Lopes T, Gardner R, Berger F, Feijó JA, Becker JD, et al. 2012. Reprogramming of DNA methylation in pollen guides epigenetic inheritance via small RNA. Cell 151: 194-205.

Cao X, Aufsatz W, Zilberman D, Mette MF, Huang MS, Matzke M, Jacobsen SE. 2003. Role of the DRM and CMT3 methyltransferases in RNAdirected DNA methylation. Curr Biol 13: 2212-2217.

Cao J, Schneeberger K, Ossowski S, Günther T, Bender S, Fitz J, Koenig D, Lanz C, Stegle O, Lippert C, et al. 2011. Whole-genome sequencing of multiple Arabidopsis thaliana populations. Nat Genet 43: 956-963.

Cingolani P, Platts A, Wang LL, Coon M, Nguyen T, Wang L, Land SJ, Lu X, Ruden DM. 2012. A program for annotating and predicting the effects of single nucleotide polymorphisms, SnpEff: SNPs in the genome of Drosophila melanogaster strain $w^{1118}$; iso-2; iso-3. Fly (Austin) 6: 80-92.

Cokus SJ, Feng S, Zhang X, Chen Z, Merriman B, Haudenschild CD, Pradhan S, Nelson SF, Pellegrini M, Jacobsen SE. 2008. Shotgun bisulphite sequencing of the Arabidopsis genome reveals DNA methylation patterning. Nature 452: 215-219.

Cubas P, Vincent C, Coen E. 1999. An epigenetic mutation responsible for natural variation in floral symmetry. Nature 401: 157-161.

Diers BW, Cary T, Thomas D, Colgrove A, Niblack T. 2010. Registration of LD00-2817P soybean germplasm line with resistance to soybean cyst nematode from PI 437654. J Plant Registrations 4: 141-144.

Dowen RH, Pelizzola M, Schmitz RJ, Lister R, Dowen JM, Nery JR, Dixon JE, Ecker JR. 2012. Widespread dynamic DNA methylation in response to biotic stress. Proc Natl Acad Sci 109: E2183-E2191.

Doyle JJ, Doyle JL, Harbison C. 2003. Chloroplast-expressed glutamine synthetase in Glycine and related Leguminosae: Phylogeny, gene duplication, and ancient polyploidy. Syst Bot 28: 567-577.

Du J, Zhong X, Bernatavichute YV, Stroud H, Feng S, Caro E, Vashisht AA, Terragni J, Chin HG, Tu A, et al. 2012. Dual binding of chromomethylase domains to H3K9me2-containing nucleosomes directs DNA methylation in plants. Cell 151: $167-180$.

Durand S, Bouche N, Perez Strand E, Loudet O, Camilleri C. 2012. Rapid establishment of genetic incompatibility through natural epigenetic variation. Curr Biol 22: 326-331.
Eichten SR, Swanson-Wagner RA, Schnable JC, Waters AJ, Hermanson PJ, Liu S, Yeh CT, Jia Y, Gendler K, Freeling M, et al. 2011. Heritable epigenetic variation among maize inbreds. PLoS Genet 7: e1002372.

Feng S, Cokus SJ, Zhang X, Chen PY, Bostick M, Goll MG, Hetzel J, Jain J, Strauss SH, Halpern ME, et al. 2010. Conservation and divergence of methylation patterning in plants and animals. Proc Natl Acad Sci 107: 8689-8694.

Gan X, Stegle O, Behr J, Steffen JG, Drewe P, Hildebrand KL, Lyngsoe R, Schultheiss SJ, Osborne EJ, Sreedharan VT, et al. 2011. Multiple reference genomes and transcriptomes for Arabidopsis thaliana. Nature 477: 419423.

Ganko EW, Meyers BC, Vision TJ. 2007. Divergence in expression between duplicated genes in Arabidopsis. Mol Biol Evol 24: 2298-2309.

Gill N, Findley S, Walling JG, Hans C, Ma J, Doyle J, Stacey G, Jackson SA. 2009. Molecular and chromosomal evidence for allopolyploidy in soybean. Plant Physiol 151: 1167-1174.

Greaves IK, Groszmann M, Ying H, Taylor JM, Peacock WJ, Dennis ES. 2012. Trans chromosomal methylation in Arabidopsis hybrids. Proc Natl Acad Sci 109: 3570-3575.

Groszmann M, Greaves IK, Albertyn ZI, Scofield GN, Peacock WJ, Dennis ES 2011. Changes in 24-nt siRNA levels in Arabidopsis hybrids suggest an epigenetic contribution to hybrid vigor. Proc Natl Acad Sci 108: 26172622.

Haas BJ, Delcher AL, Wortman JR, Salzberg SL. 2004. DAGchainer: A tool for mining segmental genome duplications and synteny. Bioinformatics 20: 3643-3646.

He G, Zhu X, Elling AA, Chen L, Wang X, Guo L, Liang M, He H, Zhang H, Chen F, et al. 2010. Global epigenetic and transcriptional trends among two rice subspecies and their reciprocal hybrids. Plant Cell 22: 17-33.

Hitchins MP, Wong JJ, Suthers G, Suter CM, Martin DI, Hawkins NJ, Ward RL. 2007. Inheritance of a cancer-associated MLH1 germ-line epimutation. $N$ Engl J Med 356: 697-705.

Huang X, Kurata N, Wei X, Wang ZX, Wang A, Zhao Q, Zhao Y, Liu K, Lu H, $\mathrm{Li} \mathrm{W}$, et al. 2012. A map of rice genome variation reveals the origin of cultivated rice. Nature 490: 497-501.

Ibarra CA, Feng X, Schoft VK, Hsieh TF, Uzawa R, Rodrigues JA, Zemach A, Chumak N, Machlicova A, Nishimura T, et al. 2012. Active DNA demethylation in plant companion cells reinforces transposon methylation in gametes. Science 337: 1360-1364.

Innes RW, Ameline-Torregrosa C, Ashfield T, Cannon E, Cannon SB, Chacko B, Chen NW, Couloux A, Dalwani A, Denny R, et al. 2008. Differential accumulation of retroelements and diversification of NB-LRR disease resistance genes in duplicated regions following polyploidy in the ancestor of soybean. Plant Physiol 148: 1740-1759.

Johannes F, Porcher E, Teixeira FK, Saliba-Colombani V, Simon M, Agier N, Bulski A, Albuisson J, Heredia F, Audigier P, et al. 2009. Assessing the impact of transgenerational epigenetic variation on complex traits. PLoS Genet 5: e1000530.

Johnson DB, Wang C, Xu J, Schultz MD, Schmitz RJ, Ecker JR, Wang L. 2012. Release factor one is nonessential in Escherichia coli. ACS Chem Biol 7: 1337-1344.

Kim M, Hyten DL, Niblack TL, Diers BW. 2011. Stacking resistance alleles from wild and domestic soybean sources improves soybean cyst nematode resistance. Crop Sci 51: 934-943.

Lam HM, Xu X, Liu X, Chen W, Yang G, Wong FL, Li MW, He W, Qin N, Wang B, et al. 2010. Resequencing of 31 wild and cultivated soybean genomes identifies patterns of genetic diversity and selection. Nat Genet 42: 1053-1059.

Langmead B, Trapnell C, Pop M, Salzberg SL. 2009. Ultrafast and memoryefficient alignment of short DNA sequences to the human genome. Genome Biol 10: R25.

Law JA, Jacobsen SE. 2010. Establishing, maintaining and modifying DNA methylation patterns in plants and animals. Nat Rev Genet 11: 204-220.

Li H, Durbin R. 2009. Fast and accurate short read alignment with BurrowsWheeler transform. Bioinformatics 25: 1754-1760.

Li H, Freeling M, Lisch D. 2010. Epigenetic reprogramming during vegetative phase change in maize. Proc Natl Acad Sci 107: 2218422189.

Libault M, Farmer A, Joshi T, Takahashi K, Langley RJ, Franklin LD, He J, Xu D, May G, Stacey G. 2010. An integrated transcriptome atlas of the crop model Glycine max, and its use in comparative analyses in plants. Plant J 63: 86-99.

Lister R, O'Malley RC, Tonti-Filippini J, Gregory BD, Berry CC, Millar AH, Ecker JR. 2008. Highly integrated single-base resolution maps of the epigenome in Arabidopsis. Cell 133: 523-536.

Lister R, Pelizzola M, Kida YS, Hawkins RD, Nery JR, Hon G, AntosiewiczBourget J, O'Malley R, Castanon R, Klugman S, et al. 2011. Hotspots of aberrant epigenomic reprogramming in human induced pluripotent stem cells. Nature 471: $68-73$.

Makarevitch I, Eichten SR, Briskine R, Waters AJ, Danilevskaya ON, Meeley RB, Myers CL, Vaughn MW, Springer NM. 2013. Genomic distribution 
of maize facultative heterochromatin marked by trimethylation of H3K27. Plant Cell 25: 780-793.

Manning K, Tör M, Poole M, Hong Y, Thompson AJ, King GJ, Giovannoni JJ, Seymour GB. 2006. A naturally occurring epigenetic mutation in a gene encoding an SBP-box transcription factor inhibits tomato fruit ripening. Nat Genet 38: 948-952.

Martínez G, Slotkin RK. 2012. Developmental relaxation of transposable element silencing in plants: Functional or byproduct? Curr Opin Plant Biol 15: 496-502.

Mathieu O, Reinders J, Caikovski M, Smathajitt C, Paszkowski J. 2007. Transgenerational stability of the Arabidopsis epigenome is coordinated by CG methylation. Cell 130: 851-862.

Ohtsu K, Smith MB, Emrich SJ, Borsuk LA, Zhou R, Chen T, Zhang X, Timmermans MC, Beck J, Buckner B, et al. 2007. Global gene expression analysis of the shoot apical meristem of maize (Zea mays L.). Plant J 52 391-404.

Ossowski S, Schneeberger K, Clark R, Lanz C, Warthmann N, Weigel D. 2008. Sequencing of natural strains of Arabidopsis thaliana with short reads. Genome Res 18: 2024-2033.

Ossowski S, Schneeberger K, Lucas-Lledó JI, Warthmann N, Clark RM, Shaw RG, Weigel D, Lynch M. 2010. The rate and molecular spectrum of spontaneous mutations in Arabidopsis thaliana. Science 327: 92-94.

Patterson GI, Thorpe CJ, Chandler VL. 1993. Paramutation, an allelic interaction, is associated with a stable and heritable reduction of transcription of the maize $b$ regulatory gene. Genetics 135: 881-894.

Perkins W, Tygert M, Ward R. 2011. $\chi 2$ and classical exact tests often wildly misreport significance; the remedy lies in computers. http://arxiv.org/ abs/1108.4126.

Rangwala SH, Elumalai R, Vanier C, Ozkan H, Galbraith DW, Richards EJ. 2006. Meiotically stable natural epialleles of Sadhu, a novel Arabidopsis retroposon. PLoS Genet 2: e36.

Reinders J, Wulff BB, Mirouze M, Mari-Ordóñez A, Dapp M, Rozhon W, Bucher E, Theiler G, Paszkowski J. 2009. Compromised stability of DNA methylation and transposon immobilization in mosaic Arabidopsis epigenomes. Genes Dev 23: 939-950.

Richards EJ. 2006. Inherited epigenetic variation—revisiting soft inheritance. Nat Rev Genet 7: 395-401.

Ronemus MJ, Galbiati M, Ticknor C, Chen J, Dellaporta SL. 1996. Demethylation-induced developmental pleiotropy in Arabidopsis. Science 273: 654-657.

Roulin A, Auer PL, Libault M, Schlueter J, Farmer A, May G, Stacey G, Doerge RW, Jackson SA. 2012. The fate of duplicated genes in a polyploid plant genome. Plant I 73: 143-153.

Roux F, Colomé-Tatché M, Edelist C, Wardenaar R, Guerche P, Hospital F, Colot V, Jansen RC, Johannes F. 2011. Genome-wide epigenetic perturbation jump-starts patterns of heritable variation found in nature. Genetics 188: 1015-1017.

Schmitz RJ, Ecker JR. 2012. Epigenetic and epigenomic variation in Arabidopsis thaliana. Trends Plant Sci 17: 149-154.

Schmitz RJ, Zhang X. 2011. High-throughput approaches for plant epigenomic studies. Curr Opin Plant Biol 14: 130-136.

Schmitz RJ, Schultz MD, Lewsey MG, O'Malley RC, Urich MA, Libiger O, Schork NJ, Ecker JR. 2011. Transgenerational epigenetic instability is a source of novel methylation variants. Science 334: 369-373

Schmitz RJ, Schultz MD, Urich MA, Nery JR, Pelizzola M, Libiger O, Alix A, McCosh RB, Chen H, Schork NJ, et al. 2013. Patterns of population epigenomic diversity. Nature 495: 193-198.

Schmutz J, Cannon SB, Schlueter J, Ma J, Mitros T, Nelson W, Hyten DL, Song Q, Thelen JJ, Cheng J, et al. 2010. Genome sequence of the palaeopolyploid soybean. Nature 463: 178-183.

Schultz MD, Schmitz RJ, Ecker JR. 2012. "Leveling" the playing field for analyses of single-base resolution DNA methylomes. Trends Genet 28: 583-585.
Shaw RG, Byers DL, Darmo E. 2000. Spontaneous mutational effects on reproductive traits of Arabidopsis thaliana. Genetics 155: 369-378.

Shen H, He H, Li J, Chen W, Wang X, Guo L, Peng Z, He G, Zhong S, Qi Y, et al. 2012. Genome-wide analysis of DNA methylation and gene expression changes in two Arabidopsis ecotypes and their reciprocal hybrids. Plant Cell 24: $875-892$.

Silveira AB, Trontin C, Cortijo S, Barau J, Del Bem LE, Loudet O, Colot V, Vincentz M. 2013. Extensive natural epigenetic variation at a de novo originated gene. PLoS Genet 9: e1003437.

Slotkin RK, Vaughn M, Borges F, Tanurdzić M, Becker JD, Feijó JA, Martienssen RA. 2009. Epigenetic reprogramming and small RNA silencing of transposable elements in pollen. Cell 136: 461-472.

Stefanovic S, Pfeil BE, Palmer JD, Doyle JJ. 2009. Relationships among phaseoloid legumes based on sequences from eight chloroplast regions. Syst Bot 34: 115-128.

Straub SC, Pfeil BE, Doyle JJ. 2006. Testing the polyploid past of soybean using a low-copy nuclear gene-is Glycine (Fabaceae: Papilionoideae) an auto- or allopolyploid? Mol Phylogenet Evol 39: 580-584.

Takuno S, Gaut BS. 2013. Gene body methylation is conserved between plant orthologs and is of evolutionary consequence. Proc Natl Acad Sci 110: $1797-1802$.

Teixeira FK, Heredia F, Sarazin A, Roudier F, Boccara M, Ciaudo C, Cruaud C, Poulain J, Berdasco M, Fraga MF, et al. 2009. A role for RNAi in the selective correction of DNA methylation defects. Science 323: 16001604

Trapnell C, Pachter L, Salzberg SL. 2009. TopHat: Discovering splice junctions with RNA-Seq. Bioinformatics 25: 1105-1111.

Trapnell C, Williams BA, Pertea G, Mortazavi A, Kwan G, van Baren MJ, Salzberg SL, Wold BJ, Pachter L. 2010. Transcript assembly and quantification by RNA-Seq reveals unannotated transcripts and isoform switching during cell differentiation. Nat Biotechnol 28: 511-515.

Tuteja JH, Zabala G, Varala K, Hudson M, Vodkin LO. 2009. Endogenous, tissue-specific short interfering RNAs silence the chalcone synthase gene family in Glycine max seed coats. Plant Cell 21: 3063-3077.

Vaughn MW, Tanurdzić M, Lippman Z, Jiang H, Carrasquillo R, Rabinowicz PD, Dedhia N, McCombie WR, Agier N, Bulski A, et al. 2007. Epigenetic natural variation in Arabidopsis thaliana. PLoS Biol 5: e174

Weigel D, Colot V. 2012. Epialleles in plant evolution. Genome Biol 13: 249 .

Widman N, Jacobsen SE, Pellegrini M. 2009. Determining the conservation of DNA methylation in Arabidopsis. Epigenetics 4: 119-124.

Woo HR, Pontes O, Pikaard CS, Richards EJ. 2007. VIM1, a methylcytosinebinding protein required for centromeric heterochromatinization. Genes Dev 21: 267-277.

Yang Z. 2007. PAML 4: Phylogenetic analysis by maximum likelihood. Mol Biol Evol 24: 1586-1591.

Yu A, Lepère G, Jay F, Wang J, Bapaume L, Wang Y, Abraham AL, Penterman J, Fischer RL, Voinnet O, et al. 2013. Dynamics and biological relevance of DNA demethylation in Arabidopsis antibacterial defense. Proc Natl Acad Sci 110: 2389-2394.

Zemach A, Kim MY, Silva P, Rodrigues JA, Dotson B, Brooks MD, Zilberman D. 2010a. Local DNA hypomethylation activates genes in rice endosperm. Proc Natl Acad Sci 107: 18729-18734.

Zemach A, McDaniel IE, Silva P, Zilberman D. 2010b. Genome-wide evolutionary analysis of eukaryotic DNA methylation. Science 328: 916919.

Zhang X, Shiu SH, Cal A, Borevitz JO. 2008. Global analysis of genetic, epigenetic and transcriptional polymorphisms in Arabidopsis thaliana using whole genome tiling arrays. PLoS Genet 4: e1000032.

Received November 22, 2012; accepted in revised form June 5, 2013. 


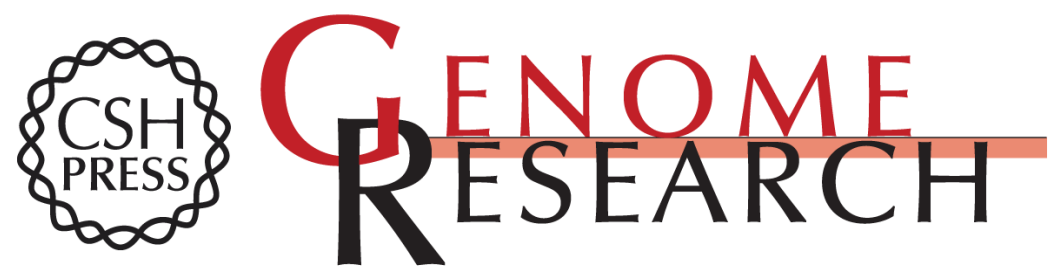

\section{Epigenome-wide inheritance of cytosine methylation variants in a recombinant inbred population}

Robert J. Schmitz, Yupeng He, Oswaldo Valdés-López, et al.

Genome Res. 2013 23: 1663-1674 originally published online June 5, 2013

Access the most recent version at doi:10.1101/gr.152538.112

\section{Supplemental http://genome.cshlp.org/content/suppl/2013/07/19/gr.152538.112.DC1 \\ Material}

Related Content $\mathrm{CHH}$ islands: de novo DNA methylation in near-gene chromatin regulation in maize Jonathan I. Gent, Nathanael A. Ellis, Lin Guo, et al.

Genome Res. April , 2013 23: 628-637 The maize methylome influences mRNA splice sites and reveals widespread paramutation-like switches guided by small RNA

Michael Regulski, Zhenyuan Lu, Jude Kendall, et al.

Genome Res. October, 2013 23: 1651-1662

References This article cites 82 articles, 26 of which can be accessed free at: http://genome.cshlp.org/content/23/10/1663.full.html\#ref-list-1

Articles cited in:

http://genome.cshlp.org/content/23/10/1663.full.html\#related-urls

Creative This article is distributed exclusively by Cold Spring Harbor Laboratory Press for the Commons License first six months after the full-issue publication date (see http://genome.cshlp.org/site/misc/terms.xhtml). After six months, it is available under a Creative Commons License (Attribution-NonCommercial 3.0 Unported), as described at http://creativecommons.org/licenses/by-nc/3.0/.

Email Alerting Receive free email alerts when new articles cite this article - sign up in the box at the Service top right corner of the article or click here.

\section{Affordable, Accurate Sequencing.} gencove

To subscribe to Genome Research go to: https://genome.cshlp.org/subscriptions 